Check for updates

Cite this: RSC Adv., 2018, 8, 23748

\title{
Administration of raloxifene hydrochloride nanosuspensions partially attenuates bone loss in ovariectomized mice
}

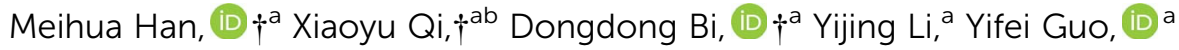 \\ Xiangtao Wang ${ }^{\star a}$ and Li Feng ${ }^{\star c}$
}

Postmenopausal osteoporosis is a systemic skeletal disease of fragility fractures due to the loss of the mass and the deterioration of the microarchitecture of bone. This study aimed to assess the effects of raloxifene hydrochloride nanosuspensions (RLX-NSps) on ovariectomized (OVX)-induced osteoporotic rats, and the underlying mechanisms were also investigated in vivo and ex vivo. RLX-NSps were successfully prepared, and the obtained RLX-NSps had a mean particle size of (91.17 \pm 0.73$) \mathrm{nm}$, PDI value of $0.201 \pm 0.03$ and zeta potential of $(36.3 \pm 1.8) \mathrm{mV}$. RLX-NSps showed a clear colloidal solution with light yellow opalescence. RLX-NSps were stable in artificial intestinal fluid, artificial gastric fluid, PBS, isotonic glucose and physiological saline. The OVX mice were administered an RLX-NSps or RLX solution for 3 weeks. The bone micro-tomographic histomorphometry and bone mineral density (BMD) were assessed by micro$\mathrm{CT}$, and the biochemical markers procollagen type I N-terminal propeptide (P1NP) and beta-isomerized C-telopeptide ( $\beta$-CTX) were determined from serum. Finally, primary bone marrow stromal cells (BMSCs) were isolated from the tibia and cultured to evaluate cell proliferation and osteogenic differentiation. The results demonstrated that the RLX-NSp group had a better effect on the bone microarchitecture than the RLX solution group. Therefore, RLX-NSps could partially attenuate bone loss more effectively than RLX solution in OVX mice by inhibiting bone resorption and improving the ability of BMSCs to proliferate and their osteogenic differentiation to some extent. Based on these results, nanosuspensions (NSps) may be a promising delivery system for postmenopausal osteoporosis therapy.

Received 23rd March 2018
Accepted 19th June 2018
DOI: $10.1039 /$ c8ra02535e
rsc.li/rsc-advances

\section{Introduction}

Postmenopausal osteoporosis is a relatively common metabolic bone disease that leads to increasing skeletal fragility and the risk of fractures due to rapid bone loss..$^{1-3}$ Over half of adults over the age of 50 are diagnosed with osteoporosis, and among them, most women have postmenopausal osteoporosis. ${ }^{4,5}$ They can be affected with osteoporotic fractures throughout their remaining lifetime. ${ }^{6}$

There are two major therapeutic approaches for osteoporosis. One is anabolic agents that function by means of improving bone formation, such as parathyroid hormone, ${ }^{7}$ and the other is antiresorptive agents, which function by means of inhibiting osteoclast-mediated bone resorption, such as

${ }^{a}$ Institute of Medicinal Plant Development, Chinese Academy of Medical Sciences, Peking Union Medical College, No. 151, Malianwa North Road, Haidian District, Beijing 100193, China. E-mail: xtaowang@163.com; Tel: +861057833266

${ }^{b}$ School of Pharmacy, Heilongjiang University of Chinese Medicine, Harbin 150040, China

'National Cancer Center/National Clinical Research Center for Cancer/Cancer Hospital, Chinese Academy of Medical Sciences and Peking Union Medical College, Beijing, 100021, China. E-mail: fengli663@126.com

$\dagger$ The first three authors contributed equally to this paper. bisphosphonates. ${ }^{8}$ Estrogen deficiency, which is related to the loss of ovarian function, leads to bone loss and is a marker of osteoporosis as a result of excessive bone resorption. ${ }^{9-11}$ To prevent postmenopausal bone loss, estrogen replacement therapy (ERT) and hormone replacement therapy (HRT) have been applied..$^{12}$ It was reported that it can increase the risk of endometrial cancer and is slightly related to an increased risk of breast cancer after using estrogen for a long time. ${ }^{13,14}$ In contrast with estrogen, many studies have demonstrated that RLX has no side effects in terms of the stimulatory effect on the endometrium and the breast. ${ }^{15-17}$

Raloxifene hydrochloride (RLX), one of the secondgeneration selective estrogen receptor modulators (SERMs), belongs to a group of benzothiophene compounds. RLX has been pushed to the market and used for preventing and treating postmenopausal osteoporosis in the clinic for a decade, ${ }^{18}$ and it has been reported that RLX was the only one of the SERMs that is viable in terms of treating and preventing osteoporotic fractures in the market in the US and the EU. ${ }^{19,20}$ Moreover, the effects have been verified in clinical trials, and RLX can increase the bone mineral density (BMD) and decrease the risk of vertebral fractures. ${ }^{21}$ 
RLX is administered in the clinic by means of oral administration. However, RLX is part of the class II category of drugs, according to the standards of the biopharmaceutical classification system (BCS) classification on account of its poor solubility. ${ }^{22}$ The dissolution rate has an influence on the bioavailability of poorly water-soluble drugs. ${ }^{23}$ The bioavailability of RLX was only $2 \%$ due to its poor aqueous solubility, and extensive first pass metabolism in humans is made by glucuronide conjugation. ${ }^{24,25}$ Although other routes for administration were effective, oral administration was still a convenient and preferable route among the various routes. ${ }^{26}$ Therefore, it is necessary to improve RLX solubility. Nanosuspensions (NSps) make it possible to solve the problem of poor solubility of some drugs, to improve therapeutic efficacy. ${ }^{27}$ As a consequence, an NSps drug delivery system may be of benefit to RLX.

In this study, we have first prepared RLX-NSps and then assessed the effect of RLX-NSps on attenuating bone loss in ovariectomized (OVX) mice and made a preliminary investigation of the underlying mechanism.

\section{Materials and methods}

\subsection{Reagents and animals}

$\alpha$-Modified Eagle's medium ( $\alpha$-MEM), fetal bovine serum (FBS) and penicillin-streptomycin $\left(5000 \mathrm{U} \mathrm{mL}^{-1}\right.$ penicillin; $5000 \mu \mathrm{g}$ $\mathrm{mL}^{-1}$ streptomycin) were purchased from GIBCO Laboratories (Grand Island, NY, USA). Triton(r) X-100 bioxtra, $\beta$-glycerophosphate disodium salt hydrate, L-ascorbic acid 2-phosphate

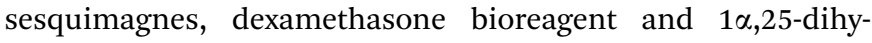
droxyvitamin D3 were purchased from Sigma-Aldrich (St. Louis, MO, USA). RLX was purchased from Wuhan Dinghui Chemical Company Ltd. Soybean lecithin (SPC) was obtained from Guangzhou Hanfang Pharmaceutical Co. Ltd. Monomethoxypoly(ethylene glycol)-poly ( $\varepsilon$-caprolactone) ( $\mathrm{MPEG}_{2000^{-}}$ $\mathrm{PCL}_{2000}$ ) was obtained from Jinan Daigang Biomaterial Co. Ltd. D-Alpha tocopherol acid polyethylene glycol succinate (TPGS) was supplied by Xi'an Healthful Biotechnology Co. Ltd. The alkaline phosphatase (ALP) kit was purchased from Nanjing Jiancheng Chemical Industrial Co. Ltd. The procollagen type I N-terminal propeptide (P1NP kit), beta-isomerized Ctelopeptide ( $\beta$-CTX) and bicinchoninic acid (BCA) kit were obtained from ELISA (IDS, Frankfurt, Germany).

The following instruments and equipment were used in these studies: Ultrasonic cleaner (Kun Shan Ultrasonic Instruments Co. Ltd., China); Dynamic Light Scattering (DLS, Zetasizer Nano ZS, Malvern Instruments, Malvern, UK); JEM-1400 electron microscope (JEOL, Tokyo, Japan); High-performance liquid chromatography (Dionex Ultimate 3000, USA); Symmetry C18 column ( $4.6 \mathrm{~mm} \times 250 \mathrm{~mm}, 5 \mu \mathrm{m}$, Waters, USA).

All animal experiments were performed on the basis of the guidelines for Ethical and Regulatory for Animal Experiments as defined by the Institute of Medicinal Plant Development (IMPLAD), Beijing, People's Republic of China. All animal experiments were also approved by the ethics committee of the IMPLAD (China) (approval number: SLXD-2016081019). The mice were permitted water ad libitum and a maintenance diet under a $12 \mathrm{~h}$ light-dark cycle at a constant room temperature $\left(22 \pm 3{ }^{\circ} \mathrm{C}\right)$ and humidity $(50 \% \pm 20 \%)$ during the experimental period.

\subsection{Chromatographic conditions for HPLC}

The concentration of the RLX drug was determined using HPLC equipped with an autosampler and with a chromatographic separation column (symmetry C18 column, $2.5 \times 4.6 \mathrm{~cm}$ ). The mobile phase was made up of $20 \mathrm{mM}$ ammonium acetate buffer ( $\mathrm{pH}$ adjusted to 4.5 with glacial acetic acid) and acetonitrile $(67: 33 \mathrm{v} / \mathrm{v})$, and the flow rate was $1 \mathrm{~mL} \mathrm{~min}^{-1}$. The detection wavelength for RLX was $288 \mathrm{~nm}$.

\subsection{Preparation of solution}

RLX solution was prepared by dissolving in $0.1 \%$ DMSO, followed by addition to $98.9 \%$ normal saline with $1 \%$ Tween $80 .^{28}$ Artificial intestinal fluid containing 1\% trypsin at $\mathrm{pH} 6.8$ in phosphate buffer saline was prepared, and the artificial gastric fluid contained $1 \%$ pepsinum in $1 \mathrm{~mol} \mathrm{~L}^{-1}$ diluted $\mathrm{HCl}^{27}$

\subsection{Preparation of RLX-NSps}

RLX-NSps were prepared by means of the anti solvent precipitation method. Briefly, RLX bulk powder was dissolved in dimethyl sulfoxide (DMSO) and a stabilizer (SPC, TPGS and $\mathrm{mPEG}_{2000}-\mathrm{PCL}_{2000}$ ) was dissolved in organic solvent; then, the two solutions were mixed, forming a mixed solution containing $12.5 \mathrm{mg} \mathrm{mL} \mathrm{mL}^{-1} \mathrm{RLX}$ with the aid of ultrasonic agitation. Next, $0.4 \mathrm{~mL}$ of the mixed solution was injected into $5 \mathrm{~mL}$ of distilled water by dropping along with ultrasonication at $250 \mathrm{~W}$ for 15 minutes. Finally, removal of the organic solvent and DMSO was carried out. Briefly, the solution containing the organic solvent was placed in a dialysis bag in a beaker with continuous stirring for 4 hours and distilled water was replaced every hour until no organic solvent remained, yielding RLX-NSps (drug : stabilizer $=1: 2$, weight ratio). Different drug-stabilizer ratios were tried for the best stabilizing effect.

\subsection{Size and morphology of RLX-NSps}

To measure the mean particle size, the polydispersity index (PDI) and the zeta potential of RLX-NSps were carried out using dynamic light scattering at $25{ }^{\circ} \mathrm{C}$. The samples of RLX-NSps were measured in triplicate. All data are shown as the mean \pm standard deviation (SD). The morphology of RLX-NSps was observed using a JEM-1400 transmission electron microscope by means of negative staining with $2 \%(\mathrm{w} / \mathrm{v})$ uranyl acetate.

\subsection{Stability of RLX-NSps in various physiological solutions}

To study whether there was an interaction that may be induced by nanoparticle aggregation under physiological conditions, a series of in vitro stability experiments were undertaken. RLXNSps were mixed with phosphate buffered solution (PBS), $1.8 \% \mathrm{NaCl}$ and $10 \%$ glucose $(1: 1, \mathrm{v} / \mathrm{v})$ prepared as an isotonic solution, followed by incubation at $37{ }^{\circ} \mathrm{C}$. At certain time intervals, measurement and analysis for changes in the mean 
particle size and particle distribution were carried out using $1 \mathrm{~mL}$ of sample in triplicate.

To evaluate the suitability of RLX-NSps for oral administration, RLX-NSps were mixed $(1: 4, \mathrm{v} / \mathrm{v})$ with artificial intestinal fluid and artificial gastric fluid and incubated at $37^{\circ} \mathrm{C}$ and then measured for particle size and particle distribution at specific time intervals. Each sample was measured in triplicate.

\subsection{Pharmacodynamic study}

A classic animal model was used to mimic postmenopausal osteoporosis through removing the ovaries of mice. ${ }^{29}$ The mice in the sham and OVX groups were treated with $0.9 \%$ normal saline vehicle daily. The mice were treated with penicillin sodium by intramuscular injection daily at 1 week post-surgery. The general conditions of the mice were observed daily, and the body weight of mice was measured weekly. The mice were given an oral administration by intragastric administration (i.g.) 12 weeks post-surgery.

Four-week-old female C57BL/6 mice ( $n=36$, weighing $20 \pm 1$ g) were obtained from the Academy of Military Medical Sciences Laboratory Animal Center (Beijing, China) and randomly divided into 4 groups with 9 mice each: (1) Sham: sham surgery; (2) OVX: the mice were bilaterally ovariectomized under anesthesia; (3) RLX solution: OVX mice were treated i.g. with $10 \mathrm{mg}$ $\mathrm{kg}^{-1}$ body weight (BW) RLX solution once every day after OVX surgery; ${ }^{30}$ and (4) RLX-NSps: OVX mice were treated i.g. with $10 \mathrm{mg} \mathrm{kg}{ }^{-1}$ BW RLX-NSps once daily after OVX surgery (RLXNSps were dissolved in nine-fold volumes of acetonitrile for the disintegration of NSps and then were filtered through a 0.22 $\mu \mathrm{m}$ filter before HPLC analysis).

After 3 weeks of oral administration, 9 mice from each group were anesthetized with pentobarbital and exsanguinated before euthanasia. Serum was separated and frozen at $-80{ }^{\circ} \mathrm{C}$ until analysis. After euthanasia, the bilateral tibias from each animal were thoroughly dissected free from soft tissue. The right tibia from each animal was fixed in $10 \%$ neutral-buffered formalin and later used for micro computed tomography (micro-CT) and histological slicing. The left tibia from each animal was used for ex vivo cell culture.

\subsection{Determination of bone BMD and microtomographic histomorphometry by micro-CT}

To evaluate the mass and microarchitecture of bone among the 4 groups, micro-CT was undertaken using an Inveon MM system (Siemens, Munich, Germany) as previously described. ${ }^{31}$ Three-dimensional images of each proximal tibia were acquired at an effective pixel size of $8.82 \mu \mathrm{m}$, and the system parameters were a voltage of $80 \mathrm{kV}$, a current of $500 \mu \mathrm{A}$, and an exposure time of $1500 \mathrm{~ms}$ for each of the 360 rotational steps. ${ }^{31}$

Parameters were calculated using an Inveon Research Workplace (Siemens) as follows: bone volume/total volume (BV/ TV) and bone surface area/bone volume (BS/BV) were used in order to evaluate the bone mass of the samples; mean trabecular thickness (Tb.Th) was determined by the local thickness at each voxel representing the trabecular meshwork; trabecular number (Tb.N) was the number of times that trabeculae crossed the complete length in a random selection across the bone volume analyzed and was calculated from the inverse of the mean distance between the middle axes of the bone structure; trabecular separation (Tb.Sp) was determined by the direct thickness of the non-bone parts; BMD in the trabecular region (0.5-1 $\mathrm{mm}$ distal to the proximal epiphysis) was determined according to the guidelines formulated by the American Society for Bone and Mineral Research. ${ }^{31}$ The linear equation of the BMD was $y=3.756 x-4190\left(R^{2}=0.999\right)$.

After being fixed in a $4 \%$ paraformaldehyde solution for 24 hours, the tibia were decalcified using $14.5 \%$ ethylenediamine tetra-acetic acid buffer $(\mathrm{pH}, 7.2)$ at room temperature for 15 days. The decalcified conditions of the samples were observed every day. The samples were embedded in paraffin and cut with a microtome (Leica, Wetzlar, Germany) into $5 \mu \mathrm{m}$ sections through the coronal plane for hematoxylin and eosin staining (HE staining).

\subsection{Assay of biochemical markers in serum}

To detect the biochemical markers in serum, blood samples were collected from the orbits of mice. Blood samples were collected into plastic tubes and centrifuged for $5 \mathrm{~min}$ at $5000 \mathrm{rpm}$. Then, the serum samples were separated and transferred to new tubes. All serum samples were stored at $-80{ }^{\circ} \mathrm{C}$ until assay. The serum levels of P1NP and $\beta$-CTX were measured using the relevant enzyme-linked immuno sorbent assay kits according to the manufacturer's protocols. Samples were measured at least in duplicate.

\subsection{Bone marrow stromal cell (BMSC) isolation, culture and osteogenic differentiation}

Generally, two ends of the femur and tibia were cut off with a rongeur, and the marrow was flushed with $1 \mathrm{~mL}$ of $\alpha$-MEM by inserting a syringe needle into one end of the bone. Cells were cultured in $\alpha$-MEM medium containing $20 \% \mathrm{FBS}, 100 \mathrm{U} \mathrm{mL}^{-1}$ penicillin $\mathrm{G}$ and $100 \mathrm{mg} \mathrm{mL}^{-1}$ streptomycin in $35 \mathrm{~mm}$ culture dishes in a water-saturated atmosphere containing $5 \% \mathrm{CO}_{2}$ at $37{ }^{\circ} \mathrm{C}$. The culture medium was replaced with new $\alpha$-MEM once every 3 days. When the cells were detached upon reaching $90 \%$ confluence in a $35 \mathrm{~mm}$ culture dish, cells were divided into two $10 \mathrm{~cm}$ Petri dishes. The passage 3-5 BMSCs were used for assays.

\subsection{CCK-8 assay and alkaline phosphatase (ALP) quantification assay}

When BMSCs were cultured to logarithmic phase, they were seeded in 96-well plates $\left(8 \times 10^{3}\right.$ cells per well). There were 4 groups for each plate and 6 wells for each group. After each day in 7 days of culture, $10 \mu \mathrm{L}$ CCK-8 solution was added to each well, followed by a $2.5 \mathrm{~h}$ incubation. After that, the absorbance was measured at $450 \mathrm{~nm}$ with an enzyme calibrator.

When BMSCs were cultured to logarithmic phase, they were then seeded in 6 -well plates $\left(8 \times 10^{5}\right.$ cells per well $)$ and the culture medium was replaced with new $\alpha$-MEM with $10 \mathrm{mM} \beta$ sodium glycerophosphate $+0.05 \mathrm{M}$ ascorbic acid $+10 \mathrm{nM}$ dexamethasone (for osteogenic differentiation) once every two 
Table 1 Characterization of RLX-NSps prepared using different stabilizers and ratios of RLX/SPC (mean \pm SD, $n=3$ )

\begin{tabular}{llllll}
\hline Stabilizer & Organic solvent & RLX : stabilizer & Size $(\mathrm{nm})$ & PDI & Zeta potential (mV) \\
\hline TPGS & DMSO & $1: 2$ & $176.8 \pm 9.24$ & $0.382 \pm 0.07$ & $27.3 \pm 2.00$ \\
mPEG $_{2000}-$ PCL $_{2000}$ & DMSO & $1: 2$ & $627.1 \pm 29.87$ & $0.142 \pm 0.01$ & $-0.903 \pm 1.20$ \\
SPC & Methanol & $1: 2$ & $91.17 \pm 0.73$ & $0.201 \pm 0.03$ & $36.3 \pm 1.80$ \\
SPC & Methanol & $1: 1$ & $101.6 \pm 3.83$ & $0.279 \pm 0.03$ & $36.1 \pm 1.40$ \\
SPC & Methanol & $3: 1$ & $111.2 \pm 6.53$ & $0.299 \pm 0.05$ & $31.7 \pm 1.50$
\end{tabular}

days. After 7 days, the culture medium was removed and the cells were washed with PBS 3 times. Then, $200 \mu \mathrm{L}$ of $1 \%$ Triton $\mathrm{X}-100$ was added to each well for $10 \mathrm{~min}$ at room temperature. Thereafter, cells were scraped from 6-well plates and put in $1.5 \mathrm{~mL}$ tubes. The cells were broken using an ultrasonic cell disruptor. Finally, the cells were centrifuged for $30 \mathrm{~min}$ at $12000 \mathrm{rcf}$ at $4{ }^{\circ} \mathrm{C}$. ALP and BCA were measured using kits according to the manufacturer's protocol, and the experiments were repeated at least 3 times.

\subsection{Statistical analysis}

Data are shown as the means \pm SD. Statistical analysis was performed using SPSS v16.0 software (IBM, Armonk, NY, USA). One-way analysis of variance (ANOVA) with post hoc least significant difference (LSD) was carried out. $P$-values less than 0.05 were considered significant.

\section{Results and discussion}

\subsection{RLX-NSps was prepared by using SPC as a stabilizer}

In this study, SPC, TPGS and $\mathrm{mPEG}_{2000}-\mathrm{PCL}_{2000}$ were tried as stabilizers to prepare RLX-NSps at a drug-stabilizer ratio of $1: 2$ (weight ratio). As seen in Table 1, RLX-NSps that was prepared by using SPC as a stabilizer had relatively good results, such as the smallest mean diameter, the narrowest size distribution and the highest zeta potential. Therefore, SPC was selected as a stabilizer to prepare RLX-NSps for further study. The structure of RLX-NSps and their expected mechanism were showed in Fig. 1.

Furthermore, to investigate the effect of RLX/stabilizer ratios on the properties of nanosuspensions, different ratios of RLXNSps were prepared. The results showed that the zeta potential (absolute value) decreased with an increase in the RLX/ stabilizer ratio. However, the results of the particle size and PDI were inverse to the zeta potential. After consideration, an RLX/stabilizer ratio of $1: 2$ was chosen for subsequent applications. The transmission electron microscopy image revealed that RLX-NSps were spherical (Fig. 2).

Stabilizer plays a vital role in the preparation of nanosuspensions. They have two main roles: to help obtain a small size of nanosuspensions and to enhance the stability of the nanosuspensions. ${ }^{32}$ The average diameter of the RLX-NSps was $(91.17 \pm 0.73) \mathrm{nm}$ with a small PDI value of $0.201 \pm 0.03$. TEM observation revealed that the RLX-NSps were spherical. TEM micrographs also demonstrated that the RLX-NSps were smaller

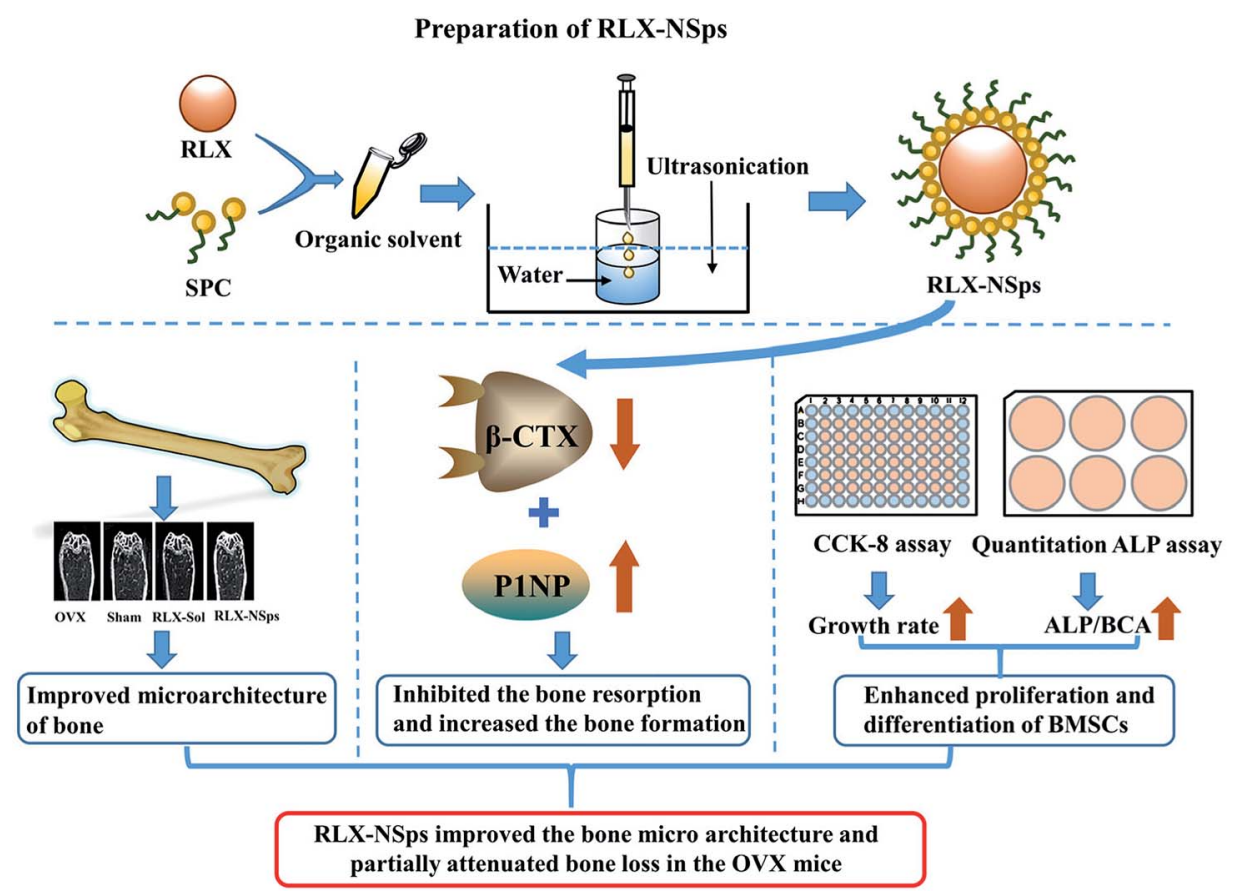

Fig. 1 Schematic representation of the preparation procedure and mechanism for RLX-NSps. 


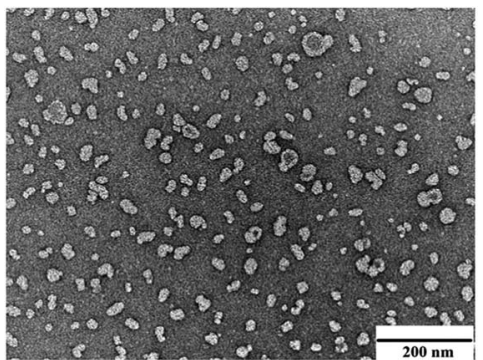

Fig. 2 TEM image of RLX-NSps. Scale bar: $200 \mathrm{~nm}$.

compared with observation by DLS examination because of the particle shrinkage from the drying process. ${ }^{27}$ RLX had poor solubility. Bulk RLX formed a turbid suspension with a sediment in water at the same concentration. However, RLX-NSps showed clear colloidal suspension with light yellow opalescence. Therefore, the solubility problem of RLX was improved.

\subsection{RLX-NSps were stable in various physiological media}

The results in Table 2 show that the particle sizes in the mixtures of RLX-NSps and isotonic glucose or physiological saline did not have big fluctuations after incubation at $37{ }^{\circ} \mathrm{C}$ for $12 \mathrm{~h}$. Moreover, the particle size in the RLX-NSps and PBS mixture was slightly increased from $0 \mathrm{~h}$ to $2 \mathrm{~h}$, but the particle size was not obviously changed after incubation at $37{ }^{\circ} \mathrm{C}$ from $2 \mathrm{~h}$ to $12 \mathrm{~h}$. Besides, there were no significant particle size enlargements during the incubation of RLX-NSps with an artificial intestinal fluid or artificial gastric fluid. RLX-NSps were stable in artificial intestinal fluid and artificial gastric fluid. These results demonstrated that RLX-NSps were suitable for oral drug delivery.

\subsection{RLX-NSps obviously improved the microarchitecture of bone in the tibia}

Bone structure researches were mainly focused on stereology research methods or computed tomography (CT) in the past time, which cannot learn the microstructure of bone. ${ }^{33,34}$ Thus, we adopted an advanced technology, micro-CT, to quantitatively analyze various parameters of bone and study the microstructure of bone. To assess the bone mass and the bone microarchitecture in 4 groups, BMD and bone morphometry were carried out by micro-CT (Fig. 3 and 4). For the analysis of the BMD and micro-CT of the proximal tibia, ex vivo analysis demonstrated that the OVX group exhibited markedly less BMD compared with the sham group $(p<0.05)$, which means that the mice indeed had induced bone loss after OVX surgery. There was no significant difference between the sham and RLX-NSps groups. The BMD of the RLX-NSps and RLX solution groups increased significantly compared with the OVX group $(p<0.01)$. Moreover, the BMD of the RLX-NSps group was higher than that of the RLX solution group ( $p<0.01)$.

The results of micro-CT analyses demonstrated that the OVX group had obviously less BV/TV, Tb.N, Tb.Th and more BS/BV, Tb.Sp than the sham group (BV/TV, Tb.N, Tb.Th, BS/BV and Tb.Sp: $p<0.01)$. Generally, consistent with the BMD results, the RLX-NSps group had a better effect on bone microarchitecture than the OVX and RLX solution groups. Of note, the RLX-NSps group had an obvious difference in the tibia microarchitecture compared with the OVX group (increased Tb.Th, BV/TV and Tb.N: $p<0.01$; decreased BS/BV and Tb.Sp: $p<0.01$ ). Moreover, the RLX-NSps group had a dramatically increased Tb.Th, BV/TV and Tb.N compared with the RLX solution group $(p<0.01)$. Additionally, the BS/BV and Tb.Sp in the RLX-NSps group were lower than that of the RLX solution group (BS/BV: $p<0.01$ and Tb.Sp: $p<0.05$ ). In addition, there was no significant difference in the BMD, BV/TV, Tb.Th, Tb.N, BS/BV, and Tb.Sp between the RLX-NSps group and the sham group. Consistent with the RLXNSps group, the RLX solution group had a markedly increased BV/TV and Tb.Th: $(p<0.05$; Tb.N: $p<0.01)$ and a significantly decreased BS/BV and Tb.Sp: $(p<0.05)$ compared with the OVX group. However, the RLX solution group had a notably increased BS/BV and Tb.Sp and a significantly decreased BV/TV, Tb.N, and Tb.Th compared with the sham group (BV/TV and Tb.N: $p<0.01$; Tb.Th, BS/BV, Tb.Sp: $p<0.05$ ).

To verify the radiologic evaluation of tibias, slices of decalcified tissues were analyzed and the results of bone morphometry from histology were similar to those of the microCT (data not shown) (Fig. 3C).

In this study, BMD, BV/TV, Tb.Th, Tb.N, BS/BV, and Tb.Sp of the RLX-NSps and RLX solution groups were all improved compared with the OVX group. This demonstrates that RLX can partially attenuate bone loss effectively in the OVX mice. Moreover, we detected that the RLX-NSps group had an obvious improvement compared with the RLX solution group in terms of the BMD, BV/TV, Tb.Th, Tb.N, BS/BV and Tb.Sp. Additionally, no significant difference was observed between the RLX-NSps and sham groups based on the parameters of the bone

Table 2 Size changes of RLX-NSps after incubation with various physiological media at $37^{\circ} \mathrm{C}$ for $12 \mathrm{~h}(\mathrm{mean} \pm \mathrm{SD}, n=3$ )

\begin{tabular}{|c|c|c|c|c|c|}
\hline \multirow[b]{2}{*}{ Time (hour) } & \multicolumn{5}{|l|}{ Size (nm) } \\
\hline & $\begin{array}{l}\text { Isotonic glucose } \\
(5 \%)\end{array}$ & $\begin{array}{l}\text { Physiological } \\
\text { saline }(0.9 \%)\end{array}$ & PBS & $\begin{array}{l}\text { Artificial } \\
\text { intestinal fluid }\end{array}$ & $\begin{array}{l}\text { Artificial } \\
\text { gastric fluid }\end{array}$ \\
\hline 0 & $131.9 \pm 2.99$ & $110.6 \pm 0.82$ & $111.8 \pm 1.83$ & $128.6 \pm 4.50$ & $109.4 \pm 6.02$ \\
\hline 2 & $138.3 \pm 4.92$ & $112.0 \pm 1.27$ & $130.1 \pm 5.20$ & $134.2 \pm 3.57$ & $115.8 \pm 3.24$ \\
\hline 4 & $143.5 \pm 9.42$ & $112.9 \pm 1.21$ & $135.3 \pm 8.67$ & $146.5 \pm 0.81$ & $120.3 \pm 1.80$ \\
\hline 6 & $144.2 \pm 4.90$ & $111.2 \pm 2.81$ & $136.4 \pm 2.48$ & $159.1 \pm 3.01$ & $130.4 \pm 3.33$ \\
\hline 12 & $152.4 \pm 3.28$ & $117.1 \pm 2.53$ & $140.0 \pm 5.29$ & $160.3 \pm 2.88$ & $132.5 \pm 4.95$ \\
\hline
\end{tabular}


A
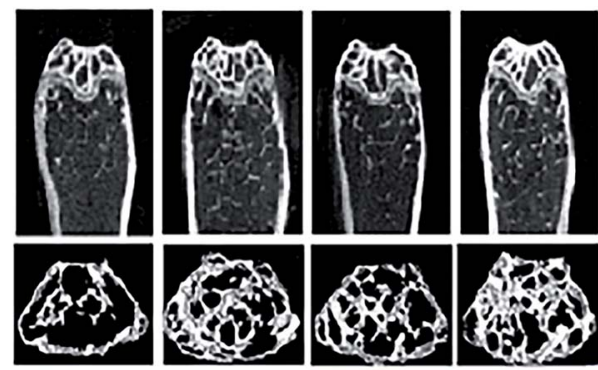

C

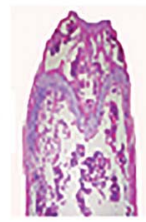

OVX

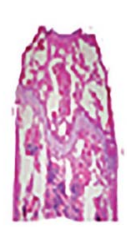

Sham

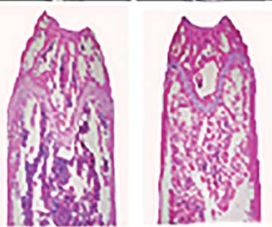

RLX-Sol RLX-NSps

Fig. 3 Representative images of changes in bone microarchitecture of the proximal tibia in mice 3 weeks after treatment. The tibias in mice were dissected and evaluated ex vivo by micro-CT and slicing of decalcified tissues (HE staining); (A) the bone microarchitecture of tibias of the four groups in the coronal plane; (B) the 3D diagram of the bone microarchitecture of tibias in the transverse plane; (C) the pictures of the slicing of decalcified tissues among the four groups (HE staining).

microtomographic histomorphometry and BMD. Based on these parameters, we deemed that administration of RLX-NSps partially attenuates bone loss in the OVX mice effectively compared with the administration an RLX solution alone. The results indicated that the efficacy of RLX in attenuating bone
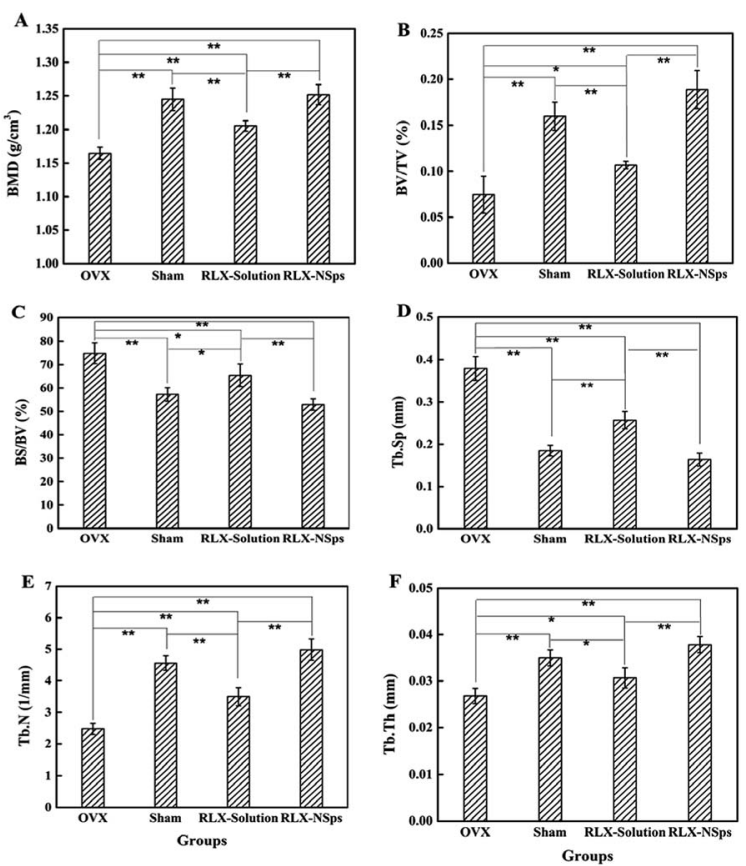

Fig. 4 The parameters of BMD (A) and bone microarchitecture (BV/TV (B), BS/BV (C), Tb.Sp (D), Tb.N (E), Tb.Th (F)) were measured in the trabecular bone of the proximal tibia (1-2 $\mathrm{mm}$ distal to the proximal epiphysis) by using micro-CT three weeks after treatment. Data are the mean $\pm \mathrm{SD}, n=9$. $* P<0.05 ; * * P<0.01$.

loss can be improved using nanotechnology. This research showed that RLX can effectively enhance BMD and reduce the risk of fractures by inhibiting bone resorption and had an effect on improving the biochemical markers connected with bone metabolism..$^{15,35}$

\subsection{RLX-NSps inhibited the bone resorption and increased the bone formation}

Data from the ELISA of P1NP and $\beta$-CTX from each group are shown in Fig. 5. $\beta$-CTX is a marker of bone resorption. The results indicated that the serum levels of $\beta$-CTX from the RLXNSps group markedly decreased compared with the OVX group (OVX group: $p<0.01$ ). The serum levels of $\beta$-CTX from the RLX-NSps group were lower than that of the RLX solution group (RLX solution group: $p<0.05$ ). However, there was no significant difference among the RLX-NSps and sham groups. Thus, the RLX-NSps group had an advantage in the serum level of $\beta$ CTX compared with the RLX solution group. Of note, the serum levels of $\beta$-CTX from the RLX solution group were significantly lower than the OVX group $(p<0.05)$. The serum levels of $\beta$-CTX from the RLX solution group were markedly increased compared with the sham group ( $p<0.05$ ) (Fig. 5A). Our study suggested that the RLX-NSps and RLX solution groups had an obvious effect on the serum level of $\beta$-CTX in OVX mice. Much research has deemed that RLX attenuates bone loss and is used to treat osteoporosis through its bone anti-resorption effect, ${ }^{36,37}$ which was consistent with our research.

P1NP is a marker of bone formation. The results indicated that serum levels of P1NP from the OVX group were obviously lower than in the sham group $(p<0.01)$. The serum levels of P1NP from the RLX-NSps group dramatically increased compared with the OVX and RLX solution groups (OVX group: $p$ $<0.01$; RLX solution group: $p<0.05$ ). Nevertheless, the serum levels of P1NP in the RLX-NSps group had no significant
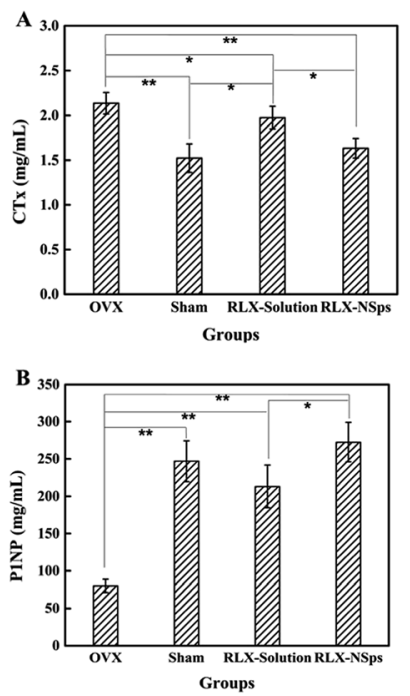

Fig. 5 Serum levels of biochemical markers of bone turnover. Serum levels of $\beta$-CTX (marker of bone resorption) (A) and P1NP (marker of bone formation) (B) 3 weeks after treatment. Data are the mean $\pm S D$ of experiments performed in triplicate. $* P<0.05$; $* * P<0.01$. 
difference compared with the sham group. Moreover, the serum levels of P1NP in the RLX solution group were dramatically higher than the OVX group (RLX group: $p<0.01$ ) (Fig. 5B). Many studies indicated that RLX treated osteoporosis through its bone anti-resorption effects. ${ }^{36,37}$ However, the results of the present study demonstrated that the RLX-NSps and RLX solution groups markedly enhanced the serum level of P1NP compared with the OVX group and that there were no significant differences compared with the sham group. Of note, the surprising part was that RLX had a certain effect on enhancing the serum level of P1NP in our study. This interesting topic merits further study.

\subsection{RLX-NSps enhanced the proliferation and differentiation of BMSCs}

The results of proliferation and differentiation of BMSCs from each group are shown in Fig. 6. To evaluate the effects on proliferation of BMSCs in the 4 groups, CCK- 8 assays were performed. The results demonstrated that there was no significant difference between each group on days 1 and 2. For days
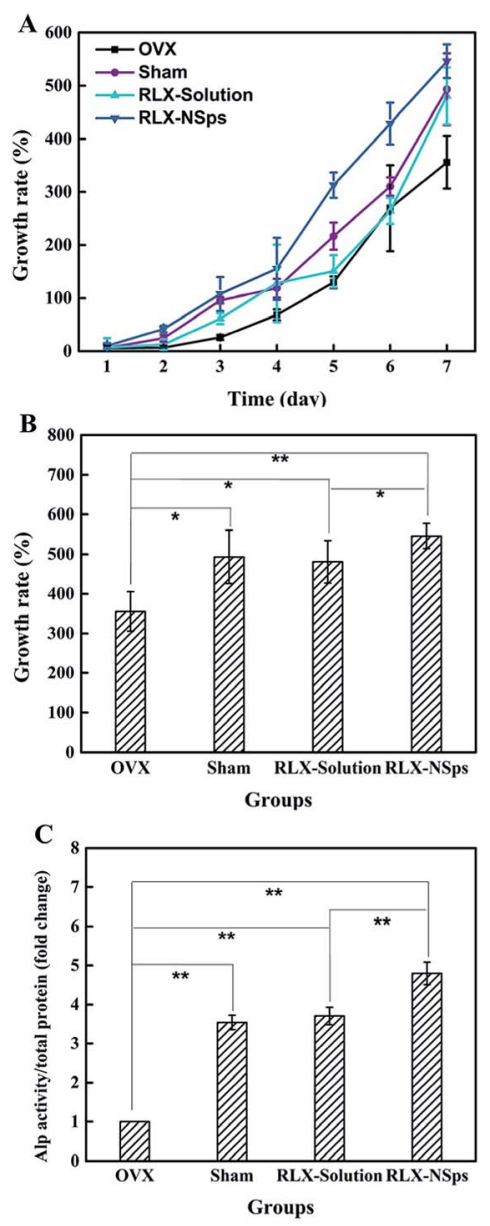

Fig. 6 The proliferation and differentiation of BMSCs ex vivo among groups. BMSC proliferation was evaluated by the CCK- 8 assay during 7 days (A). The growth rate among groups on day 7 (B). The ALP/BCA (a marker of bone formation) during osteogenic differentiation of BMSCs (C) 3 weeks after treatment. Data are means \pm SD from experiments carried out in triplicate. $* P<0.05$; $* * P<0.01$.
3-6, there were significant differences among some groups. The BMSCs from the RLX-NSps group always had a relatively fast growth during the entire process (data not shown) (Fig. 6A). On day 7, the growth rate from the RLX-NSps group significantly increased compared with the OVX and RLX solution groups (OVX group: $p<0.01$; RLX solution group: $p<0.05$ ). Nevertheless, there was no significant difference between the sham and RLX-NSps groups. Moreover, the growth rate of the RLX solution group was significantly higher than the OVX group $(p<0.05)$. However, the growth rate from the RLX solution group had no significant difference compared with the sham group. In addition, the growth rate of the OVX group was dramatically lower than the sham group $(p<0.05)$ (Fig. 6B).

To assess the effects on osteogenic differentiation in the 4 groups, the quantitative ALP assay was undertaken. The ALP/ BCA from the RLX-NSps group was higher than that of the OVX and RLX solution groups $(p<0.01)$. Nevertheless, there was no significant difference between the sham and RLX-NSps groups. Besides, the ALP/BCA of the sham and RLX solution groups dramatically increased compared with that of the OVX group ( $p<0.01$ ), but the results of the ALP/BCA from the sham and RLX solution groups had no significant difference (Fig. 6C).

BMSC proliferation and osteogenic differentiation are beneficial to bone reconstruction. ALP was secreted from the immature osteoblasts, and ALP activity is considered as an early differentiation marker. ${ }^{38}$ In our present study, the trend of the quantitation of the ALP assay was similar to that of the rate of cell growth. Therefore, RLX had an effect on enhancing bone formation to a certain degree. Interestingly, RLX not only had an advantage in inhibiting the bone resorption, but it also enhanced the bone formation for treating the osteoporosis, and the efficacy of the RLX-NSps group was superior to the RLX solution group. In this study, nano-formulation of drugs can improve the problem of poor solubility.

\section{Conclusions}

In the study, the obtained RLX-NSps showed small particle size and outstanding stability in various physiological solutions. Moreover, the RLX-NSps group had a better effect on the bone microarchitecture than the RLX solution group. RLX-NSps could partially attenuate bone loss more effectively than RLX solution in OVX mice by inhibiting the bone resorption and improving the ability of BMSC proliferation and osteogenic differentiation to some extent. Based on these results, RLXNSps may be a promising delivery system for postmenopausal osteoporosis.

\section{Conflicts of interest}

No potential conflict of interest was reported by the authors.

\section{Abbreviation list}

BMD

NSps
Bone mineral density Nanosuspensions 
OVX

Ovariectomized

$\mathrm{mPEG}_{2000}-\mathrm{PCL}_{2000}$; PEG MW 2000, PCL MW 2000

ALP

BCA

BMSCs

$\mathrm{BS} / \mathrm{BV}$

$\mathrm{BV} / \mathrm{TV}$

$\beta$-CTX

CCK-8

P1NP

RLX

SPC

Tb.N

Tb.Sp

Tb.Th

TPGS monomethoxypoly(ethylene glycol)-poly( $\varepsilon$-caprolactone) Alkaline phosphatase Bicinchoninic acid Bone marrow stromal cells Bone surface area/bone volume Bone volume/total volume Beta-isomerized C-telopeptide Cell counting kit-8 Procollagen type I N-terminal propeptide

Raloxifene hydrochloride

Soybean lecithin

Trabecular number

Trabecular separation

Trabecular thickness

D-Alpha tocopherol acid

polyethylene glycol succinate

\section{Acknowledgements}

This work was supported by the National Natural Science Foundation of China (No. U1401223) and the Beijing Municipal Science \& Technology Commission "Ten Diseases and Ten Medicine” Research Project (No. Z171100001717017).

\section{References}

1 P. Fardellone, B. Cortet, E. Legrand, X. Bresse, S. BisotLocard, A. M. Vigneron and A. Beresniak, Jt., Bone, Spine, 2010, 77, 53-57.

2 F. R. Golmohamdi, M. Abbasi, A. K. Karyani and A. A. Sari, Electron. Physician., 2016, 8, 3434-3440.

3 Y. Kameda, M. Takahata, S. Mikuni, T. Shimizu, H. Hamano, T. Angata, S. Hatakeyama, M. Kinjo and N. Iwasaki, Bone, 2015, 71, 217-226.

4 X. Luo, J. Zhang, C. Zhang, C. He and P. Wang, Disabil. Rehabil., 2017, 39, 2315-2323.

5 C. Simonelli and M. S. Burke, Curr. Med. Res. Opin., 2006, 22, 1101-1108.

6 P. Hadji, S. Klein, H. Gothe, B. Haussler, T. Kless, T. Schmidt, T. Steinle, F. Verheyen and R. Linder, Dtsch. Ärztebl. Int., 2013, 110, 52-57.

7 D. C. Bauer, ACP J. Club, 2006, 145, 71.

8 J. H. Park, E. K. Park, D. W. Koo, S. Lee, S. H. Lee, G. T. Kim and S. G. Lee, BMC Musculoskeletal Disord., 2017, 18, 0171514.

9 A. Cline-Smith, J. Gibbs, E. Shashkova, Z. S. Buchwald, D. V. Novack and R. Aurora, JCI Insight, 2016, 1, e88839.

10 O. Tabatabaei-Malazy, P. Salari, P. Khashayar and B. Larijani, Daru, J. Fac. Pharm., Tehran Univ. Med. Sci., 2017, 25, 2.

11 M. Zaidi, Nat. Med., 2007, 13, 791-801.

12 N. F. Col, M. H. Eckman, R. H. Karas, S. G. Pauker, R. J. Goldberg, E. M. Ross, R. K. Orr and J. B. Wong, JAMA, 1997, 277, 1140-1147.

13 D. Grady, T. Gebretsadik, K. Kerlikowske, V. Ernster and D. Petitti, Obstet. Gynecol., 1995, 85, 304-313.

14 J. E. Rossouw, G. L. Anderson, R. L. Prentice, A. Z. LaCroix, C. Kooperberg, M. L. Stefanick, R. D. Jackson, S. A. Beresford, B. V. Howard, K. C. Johnson, J. M. Kotchen and J. Ockene, JAMA, 2002, 288, 321-333.

15 I. A. Abdelazim, K. M. Abdelrazak, M. Al-Kadi, A. H. Yehia, B. M. Nusair and M. A. Faza, Arch. Osteoporos., 2014, 9, 189.

16 S. M. Boss, W. J. Huster, J. A. Neild, M. D. Glant, C. C. Eisenhut and M. W. Draper, Am. J. Obstet. Gynecol., 1997, 177, 1458-1464.

17 J. A. Cauley, L. Norton, M. E. Lippman, S. Eckert, K. A. Krueger, D. W. Purdie, J. Farrerons, A. Karasik, D. Mellstrom, K. W. Ng, J. J. Stepan, T. J. Powles, M. Morrow, A. Costa, S. L. Silfen, E. L. Walls, H. Schmitt, D. B. Muchmore, V. C. Jordan and L. G. Ste-Marie, Breast Cancer Res. Treat., 2001, 65, 125-134.

18 S. S. Ko and V. C. Jordan, Expert Opin. Pharmacother., 2011, 12, 657-674.

19 K. Visvanathan, R. T. Chlebowski, P. Hurley, N. F. Col, M. Ropka, D. Collyar, M. Morrow, C. Runowicz, K. I. Pritchard, K. Hagerty, B. Arun, J. Garber, V. G. Vogel, J. L. Wade, P. Brown, J. Cuzick, B. S. Kramer and S. M. Lippman, J. Clin. Oncol., 2009, 27, 3235-3258.

20 V. G. Vogel, J. P. Costantino, D. L. Wickerham, W. M. Cronin, R. S. Cecchini, J. N. Atkins, T. B. Bevers, L. Fehrenbacher, E. R. Pajon, J. L. Wade 3rd, A. Robidoux, R. G. Margolese, J. James, C. D. Runowicz, P. A. Ganz, S. E. Reis, W. McCaskill-Stevens, L. G. Ford, V. C. Jordan and N. Wolmark, Cancer Prev. Res., 2010, 3, 696-706.

21 P. D'Amelio and G. C. Isaia, Expert Opin. Pharmacother., 2013, 14, 949-956.

22 P. H. Patil, V. S. Belgamwar, P. R. Patil and S. J. Surana, J. Pharm., 2013, 2013, 527380.

23 S. Golmohammadzadeh, N. Farhadian, A. Biriaee, F. Dehghani and B. Khameneh, Drug Dev. Ind. Pharm., 2017, 43, 1619-1625.

24 D. Saini, M. Fazil, M. M. Ali, S. Baboota and J. Ali, Drug Delivery, 2015, 22, 823-836.

25 A. Velpula, R. Jukanti, K. Y. Janga, S. Sunkavalli, S. Bandari, P. Kandadi and P. R. Veerareddy, Drug Dev. Ind. Pharm., 2013, 39, 1895-1906.

26 N. V. Shah, A. K. Seth, R. Balaraman, C. J. Aundhia, R. A. Maheshwari and G. R. Parmar, J. Adv. Res., 2016, 7, 423-434.

27 J. Hong, Y. Li, Y. Xiao, Y. Li, Y. Guo, H. Kuang and X. Wang, Colloids Surf., B, 2016, 145, 319-327.

28 H. Cho, G. L. Indig, J. Weichert, H. C. Shin and G. S. Kwon, Nanomedicine, 2012, 8, 228-236.

29 Y. H. Hwang, K. J. Kim, J. J. Kim, K. Y. Kang, S. J. Lee, G. Y. Jeong, K. H. Choi, Y. J. Son and S. T. Yee, Evid.-Based Complementary Altern. Med., 2015, 2015, 373145.

30 N. Furusyo, E. Ogawa, M. Sudoh, M. Murata, T. Ihara, T. Hayashi, H. Ikezaki, S. Hiramine, H. Mukae, K. Toyoda, 
H. Taniai, K. Okada, M. Kainuma, E. Kajiwara and J. Hayashi, J. Hepatol., 2012, 57, 1186-1192.

31 H. Liu, W. Li, Y. Liu, X. Zhang and Y. Zhou, Stem Cell Res. Ther., 2015, 6, 200.

32 Y. Li, J. Hong, H. Li, X. Qi, Y. Guo, M. Han and X. Wang, Drug Delivery, 2017, 24, 1491-1500.

33 X. Wang, R. R. Zauel, D. S. Rao and D. P. Fyhrie, Bone, 2008, 42, 1184-1192.

34 W. Pistoia, B. van Rietbergen, A. Laib and P. Ruegsegger, J. Biomech. Eng., 2001, 123, 176-183.
35 H. Liu, W. Li, X. Ge, S. Jia and B. Li, Life Sci., 2016, 166, 2026.

36 J. Takada, K. Iba, T. Yoshizaki and T. Yamashita, Journal of Orthopaedic Surgery, 2012, 20, 209-213.

37 J. Takada, S. Ikeda, T. Kusanagi, S. Mizuno, H. Wada, K. Iba, T. Yoshizaki and T. Yamashita, Clinical Cases in Mineral and Bone Metabolism, 2016, 13, 25-28.

38 G. N. Bancroft, V. I. Sikavitsas, J. van den Dolder, T. L. Sheffield, C. G. Ambrose, J. A. Jansen and A. G. Mikos, Proc. Natl. Acad. Sci. U. S. A., 2002, 99, 1260012605. 